\title{
RESERVES OF MOTOR-VEHICLE INSURANCE IN FINLAND
}

\author{
by \\ Teivo Pentikäinen \\ Helsinki (Finland)
}

r. The Ministry of Social Affairs, which acts i.a. as the supervising office in Finland, has given instructions regarding the normal reserves of insurance companies. A summary of these and some comments are given here as far as they concern motor-vehicle insurance. The instructions as far as they concern the subject referred to in the following in the items 2-6, 9 and Io, were compiled by a committee, presided over by Mr. I. Ketola, M. Sc., which availed itself of the experience of several Finnish insurance companies.

In order to give a review of the system as a whole many items, which are mathematically trivial and well-known, are briefly explained.

\section{Premium reserve}

2. The conventional principle of "pro rata parte temporis" is followed, which leads to the well-known reserve

$$
V=0.4 \mathrm{P}
$$

where $P$ is the premium income of the company. This provides that the days when the premiums fall due are approximately equally distributed over the year (which can be checked from the premium sums of the different months in the book-keeping) or at least have no cluster points in the second half of the year and that the cost of the collecting of premiums is not less than $0.2 \mathrm{P}$. A more accurate calculation takes into account i.a. temporary short term policies etc.

\section{Claims reserve}

3. In casu-reserve. All unpaid claims (except those mentioned later) due to accidents which occured before the end of the account 
year, are listed and rated one by one. Doubtful cases, e.g. where the cause of the accident is still under litigation, are calculated in accordance with the "worst" alternative.

4. Unknown claims. There is always a certain time lapse between the accident and its notification to the company. The in casureserve can therefore never contain all claims, because a certain number of them are not yet known to the company when the in casu-list is made up. For these "lagging" or "unknown" claims a provision is made, based on the statistical experience of previous years.

It would be best to deduct the rules of unknown provision separately from each company's own statistics, because the notification time can vary from company to company. The statistics are, however, often too narrow, especially for small companies; nor is any significant difference to be found among the various Finnish companies. That is why the Ministry of Social Affairs has given normal rules, which may be applied by all companies, unless their own experience later on (see nr. Io later) contradicts them.

In the attached table the experience of some Finnish companies is shown for the third party motor insurance. The numbers represent the claims which were notified to the company during an observation year and classified in accordance with the year of the accident in question. The numbers are the claims in question in percen-

UNKNOWN CLAIMS IN PERCENTAGE FROM THE PREMIUMS

OF THE ORIGINAL YEAR $=q_{t-i}$

Third party motor insurance.

\begin{tabular}{|c|c|c|c|c|c|c|c|c|c|c|c|c|c|}
\hline \multirow{2}{*}{$\begin{array}{c}\text { Year } \\
\text { of the } \\
\text { acci- } \\
\text { dent }\end{array}$} & \multicolumn{11}{|c|}{$q_{t-i}$ for I I companies } & \multirow{2}{*}{$\overline{\mathrm{q}}_{i}$} & \multirow{2}{*}{$Q_{i}$} \\
\hline & I & 2 & 3 & 4 & 5 & 6 & 7 & 8 & 9 & IO & I I & & \\
\hline$t-\mathrm{I}$ & II, 7 & I6,4 & I I , 2 & 28,6 & 25,3 & 29,9 & 26,4 & $16, I$ & 13,2 & 17,0 & 14,5 & I9, I & $3^{\circ}, 5$ \\
\hline$t-2$ & 3,9 & 8,3 & 2,6 & 2,5 & 3,0 & 5,3 & 6,3 & $0, \mathrm{I}$ & 0,7 & 0,7 & 4,9 & 3,5 & 4,4 \\
\hline$t-3$ & - & 0,3 & 0,9 & 0,4 & 0,9 & 0,5 & 1,3 & - & $0, \mathbf{I}$ & $\mathbf{I}, 3$ & 0,6 & 0,5 & 0,9 \\
\hline$t-4$ & - & $0, \mathbf{I}$ & 0,2 & 0,2 & 0,2 & $\mathbf{O}, \mathbf{I}$ & $\mathrm{O}, \mathrm{I}$ & - & - & - & 0,2 & $0, I$ & 0,4 \\
\hline$t-5$ & - & - & 0,3 & - & $0, I$ & 0,2 & $0, \mathrm{I}$ & 一 & - & - & 0,7 & $0, I$ & 0,3 \\
\hline$t-6$ & - & - & - & 0,2 & 0,3 & - & - & - & - & - & 0,4 & $0, I$ & 0,2 \\
\hline$t-7$ & 一 & - & - & - & - & - & - & - & - & - & - & - & $0, \mathbf{I}$ \\
\hline$t-8$ & - & 一 & $I, O$ & 一 & 一 & - & - & - & - & - & - & $0, \mathrm{I}$ & $0, \mathbf{I}$ \\
\hline$t-9$ & - & - & - & 0,2 & - & - & - & -- & 一 & 一 & - & - & - \\
\hline
\end{tabular}


tage of the premiums of the original year. It can be seen that the lapse before notification can be rather long and that consequently a long period for statistics is needed to get an adequate basis for the provision. ${ }^{1}$ )

$t=$ year of the notification

$t-i=$ year when the accident in question happened

$P_{t-i}=$ total amount of the premiums of the year $t-i$

$q_{t-i}=$ the claims of the accident in year $t-i$ but notified in year $t$ (and paid in this year or later) in percentage from $P_{t-1}$

$\bar{q}_{t-t}=$ mean value of the different companies $q_{t-i}$

$Q_{t-1}=\sum_{i=1}^{\infty} q_{t-1}$

For the index $i=\mathrm{I}$ an empiric security margin is added.

$X=$ the provision for unknown claims. When the coefficients $Q$ are once estimated from past experience, they can then be kept as constants and applied several years for reserve calculations.

$$
\begin{aligned}
X=\sum_{i=1}^{\infty} Q_{t-i} P_{t-i} & \text { (2) } \\
=0.305 P_{t-1}+0.044 P_{t-2}+0.009 P_{t-3}+\ldots \approx 0.3 \mathrm{I} P_{t-1}+ & +0.06 P_{t-2}
\end{aligned}
$$

The provision of unknown claims depends to a certain degree on the time at which the list of known claims closes. If a company considers as known every claim which was notified at the latest by February after the account year, the provision for the unknown claims can be smaller than in a case where only, e.g., the claims notified up to December are observed. To standardize the concept and to get comparable figures a definition has been introduced in Finland so that the list of the known claims may contain only those which were notified to the company before the end of the account year. Every other claim is unknown.

5. Annuities. A reserve for annuities which occur in connection with the third party motor insurance, is calculated in accordance with conventional actuarial principles.

1) Owing to the new act concerning third party motor insurance the courts may no longer take traffic accident trials into consideration unless a certificate is obtained from the insurance company, stating whether the company accepts the claim demanded or not. This new system will probably considerably shorten the notification time and reduce the provision of the unknown claims. 
6. Return of premium. If the company is liable to return a share of the premium in case of cancellation of the insurance (the vehicle is sold or withdrawn from traffic), a provision for return is needed. It can be made in accordance with the principles explained in $\mathrm{nr} .4$.

\section{Collective methods}

7. General remarks. If the number of outstanding claims is large, the in casu-method is labourious. Very often the in casu estimation of the claims can be only approximative, which makes the result to a certain degree vague, the auditors and supervising officers have then the very difficult duty of convincing themselves that an adequate security is observed (checking methods are referred to in nr. Io later on). In such circumstances collective provisions may be advisable.

In earlier years a so called standard or surplus method was used by some companies. The provision during one or two years equalled premiums less claims and commissions paid and the in casu provision was not made until after this period. This system was, however, unsatisfactory in so as cases where the total business gave a loss was concerned, as the provision remained too small. A company which might be on an unsound basis was in this way able to conceal its weakness. That is why this method is not allowed in Finland any longer.

8. Settlement time method. The size of the claims reserve depends essentially on the average settlement time of the claims. This can be measured empirically e.g. by taking a sample of paid claims. Statistics of claim amounts $S_{i}$ and the corresponding settlement times $t_{i}$ is made up, $t_{i}$ being the time between the occurrence of the accident and the payment of the claim. If the claim is paid in several parts each of them is taken separately, for pensions the moment of settlement is the final fixing of the pension and the transferring of the capital value ot the pension fund. As is easily seen the claims reserve is then:

$$
V=\frac{C}{365} \frac{\Sigma S_{i} t_{i}}{\Sigma S_{i}}
$$

where $\mathrm{C}$ is the amount of the claims paid per annum and both sums cover the whole sample in question. The time unit for $t_{i}$ 
is a day. The reserve includes a provision both for known and unknown claims. Deducting the quotient of both the sums from several samples it is possible to get some idea of the accuracy of the method. A better stability is gained if instead of $C$ the premium income $P$ is chosen as the basis. For this purpose the rate $c=C / P$ is estimated from the statistics of several years. Then

$$
V=\frac{c}{365} \frac{\Sigma S_{i} t_{i}}{\Sigma S_{i}} \cdot P
$$

When the factor of $P$ has once been calculated, it can often be used for many years.

The formulas (3) provide that the mass of claims is fairly homogeneous and not too small. The homogeneity is reached by taking out the large claims (see number 9). The quotient of the sums in the formula is in fact the weighed mean value of the settlement time. The weighing by the sums $S_{i}$ is needed because often the large claims are more delayed than the small ones.

9. Settlement statistics method. One drawback of method number 8 is i.a. that the possible seasonal variations in the quality of claims, e.g. due to weather conditions, are not easy to take into account. For that reason the following method is used for motor vehicle insurance in Finland, even though the fixing of the necessary provision factors is more tedious and demands a longer observation period.

This method is simply based on statistics where, after a certain book-keeping year, all claims which were known but outstanding

Known property losses

\begin{tabular}{|c|c|c|c|c|c|c|c|c|c|c|c|}
\hline \multirow{2}{*}{$\begin{array}{l}\text { Year of the } \\
\text { accident }\end{array}$} & \multicolumn{10}{|c|}{$K_{t-i}$ for different companies, per cent } & \multirow{2}{*}{$\bar{K}_{t-1}$} \\
\hline & I & 2 & 3 & 4 & 5 & 6 & 7 & 8 & 9 & Io & \\
\hline I & $\mathrm{I} 7, \mathrm{I}$ & 12,4 & II, 8 & I 2,7 & 12,5 & I 6,9 & Io, 2 & r 3,4 & I9,6 & 20,9 & 14,8 \\
\hline 2 & 8,7 & 3,4 & 4,0 & 6,8 & 4,3 & 5,4 & I I,4 & 2,9 & 4,0 & 6,8 & 5,8 \\
\hline 3 & 5,6 & 2,5 & $I, 6$ & $I, 6$ & I, 7 & $I, 6$ & 4,7 & 10,4 & 2,8 & 2,5 & 3,5 \\
\hline 4 & 3.7 & 3,8 & 0,6 & 0,8 & I, O & 4,2 & 1,3 & 4,6 & 1,3 & 0,9 & 2,2 \\
\hline 5 & 2,0 & 0,6 & 2,9 & 0,4 & $\mathbf{0}, \mathbf{I}$ & - & $0, I$ & 0,5 & 1,2 & 0,6 & 0,8 \\
\hline 6 & 0,6 & - & 0,6 & 1,7 & 0,3 & - & - & - & 0,5 & $0, I$ & 0,4 \\
\hline 7 & - & - & - & - & - & - & 一 & - & - & - & - \\
\hline 8 & - & 3,0 & - & - & - & - & - & - & - & - & 0,3 \\
\hline 9 & - & 3,8 & - & - & - & - & - & - & - & - & 0,4 \\
\hline 10 & - & 0,4 & - & - & 一 & - & - & 一 & - & - & 0,0 \\
\hline
\end{tabular}


$t=$ the year next following the account year

$t-i=$ the year of the accident $(i=1,2, \ldots)$

$P_{t-1}=$ total amount of the premiums of the year $t-i$

$K_{t-i}=$ the claims, which were known already at the end of the account year $t-i$, and where the accident had happened in year $t-i$ (the year of the payment being the year $t$ or later), in percentage of the premiums $P_{t-1}$.

$R \quad=$ provision for the known claims.

at the end of the year in question are observed, when paid, and arranged in accordance with the date of the accident year. Third party motor insurance statistics of this kind are shown in the attached table.

$$
\begin{aligned}
R & =\sum_{i=1}^{\infty} K_{t-i} P_{t-i} \\
& =0.18 P_{t-1}+0.058 P_{t-2}+0.035 P_{t-3}+\ldots \\
& \approx 0.18 P_{t-1}+0.07 P_{t-2}+0.07 P_{t-3}
\end{aligned}
$$

Here in the $K_{t-1}$ a security margin is included, that is why the number 0.184 is used instead of 0.148 in the table.

From the above-mentioned formulas the following normal provisions recommended by the Ministry of Social Affairs are deducted.

Third party motor insurance:

I) Known personal injuries: in casu

2) Known large property losses: (larger than $0.05 \mathrm{P})$ in casu

3) Other known property losses: $0,18 P+0.07\left(P_{-1}+P_{-2}\right)$

4) Unknown losses: 0,3 I $P+0.06 P_{-1}$

Here $\mathrm{P}$ is the annual premium income of this branch in the account year and $\mathrm{P}_{-1}$ and $P_{-2}$ those of the previous year and the year before that.

The corresponding rules for other motor vehicle insurance is as follows: 

I) Known great losses (greater than $0.05 P$ )
2) Other known losses
in casu
3) Unknown losses
o.xI $P$
0.15P

The large losses are picked out of the collective provision to secure the necessary stability of the method. The limit $0.05 P$ depends on the size of the business in question. This improves the security margin of the small companies, which is also desirable because of the random fluctuations of the actual amount of the outstanding claims.

\section{Checking the adequacy of the provision}

Io. The adequacy of in casu provisions depends very much on the skill and trustworthiness of the rating staff. The auditors and supervising autorities, who, in general, are not specialists in such rating and who are not able to see every damaged vehicle, have great difficulty in checking the adequacy of the provisions. On the other hand also the collective methods require checking to make sure that they are suitable to the company in question and that the coefficients used are not becoming absolete. An important method. of checking these things is to make, afterwards, a calculation of the final settlement:

Surplus of the provision $=$ original provision - claims paid claims transferred to the pension fund - claims still outstanding.

The still outstanding claims can be obtained by in casu-method (known claims) or by the collective method (both still unknown and known). From the formulas (2) and (4) we can get a collective provision for the claims which are still outstanding one year after the end of the account year (= year of the original reserving):

Still unknown claims $=\sum_{t=1}^{\infty} Q_{t-i-1} P_{t-i} \approx 0.09 P_{t-1}$

(Coefficient 0.09 includes security margin, the exact number in accordance with the table on the page 2 being 0.044 )

In the collective method the known claims still outstanding are $=$

$$
\sum_{i=1}^{\infty} K_{t-i-1} P_{t-i} \approx 0.09 P_{t-2}+0.07 P_{t-2}
$$




\section{Catastrofic and excess losses}

II. As is well-known the profit $( \pm)$ of the insurance business varies from year to year considerably due to random fluctuations and variations in basic probabilities. The latter phenomenon is caused by many kinds of trends, e.g. the number of vehicles in the country, road conditions etc. have an apparent influence upon the the motor vehicle insurance branches. In addition to that the basic probabilities have periodical fluctuations depending on such circumstances as weather (slippery roads etc.) and economic conditions. If the economic conditions are favourable, there is also greater motor-vehicle activity and they are on an average more hours per day in traffic and therefore subject to greater risk. The traffic frequency is then also higher.

For all the above mentioned fluctuations every insurance company must have extra reserves in addition to the proper technical reserves (= premium reserve and claims reserve). These extra reserves can be either in the form of the company's own capital (= share capital, reserve fund etc.) or as an additional provision in the technical reserves. The conventional procedure may be, in most countries, that every company has, and must have, reserve funds to meet these excess losses but the technical reserves also include certain "security margins". These margins may, however, often be insufficient to meet the whole pressure of the fluctuations, (even if the national instructions of the supervising office allow some "tolerance" which can be increased or decreased) which compels the companies to maintain an overeffective reinsurance cover. The inconvenient taxation can also to a great degree prevent the use of the reserve funds for equalisations and the companies are perhaps unwilling to cover the loss from visible reserves because of competition. One way to prevent these drawbacks and to facilitate sound development is to change the conventional definition of the technical reserves so that also the above mentioned fluctuations are taken into account. The premium reserve and the claims reserve would then, by definition, cover the future liability of the company at a certain security level less the capital value of the future premiums (the conventional definition being technical reserves $=$ cap. value of the future liability less cap. value of the 
future premiums). This means that a "fluctuation" or ,catastrophe" reserve is included in the technical reserve to adjust the fluctuations in question.

The Finnish Insurance Company Act of the year $195^{2}$ has accepted this procedure prescribing, as a part of the conventional claims reserve, "an adjustment reserve to cover excess losses, which reserve is to be calculated in accordance with the principles of the theory of risk". This reserve functions as an equalising mechanism in the insurance business, which is realised so that the amount of the reserve has two limits and it can be adjusted from year to year between these limits. The lower limit may be zero (because every company must anyway have also normal reserve funds which guarantee the necessary solvency). The upper limit shows how far the adjustment reserve is still to be considered as a "technical reserve" (and free of taxes). An interesting duty of the actuaries is to find a proper formula for the upper limit. In addition to this a transfer rule is applied. If the total amount of loss on the company's own retention is less than normal (= average of the past I0-30 years adjusted by some trend coefficients), the surplus is transferred to the adjustment reserve. On the other hand excess loss is covered by a decrease of this reserve. This transfer-rule is not necessary from the insurance point of view, a freedom to move between the limits would be more convenient. The transfer formula is intended to prevent arbitrary (if the upper limit is not reached) changes of profit in the book-keeping and so escape taxation. At the start of the system an initial amount is allowed to be transferred to the adjustment reserve. The trend coefficients of the transfer rule allow a further growth of the reserve in the long run towards to a fairly high upper limit.

The adjustment provision has been in use since 1952 . It has considerably helped the companies to improve their solvency, to increase their net retention and to avoid unnecessary reinsurance costs.

I2. We will consider briefly the problem of fixing the size of the adjustment reserve (the aforementioned upper limit). We can define it in the well-known way by seeking an amount $U$ which, by certain security probability $\mathrm{I}-\varepsilon$, satisfies the inequalities 


$$
\left.\begin{array}{l}
U+\lambda P>y_{1} \\
U+2 \lambda P>y_{1}+y_{2} \\
----- \\
U+n \lambda>y_{1}+y_{2}+\ldots+y_{n}
\end{array}\right\}
$$

where $P$ is the net premium income in the company's own retention $y_{i}$ the loss $( \pm)$ of the $i$ : th year and $\lambda$ the security margin. In the theory of risk numerous solutions are known for this problem. Because this system is used by all the companies in the country, many of which have no actuary or other mathematically qualified person in service, the rules applied must be very simple. Consequently the most difficult task is to find proper approximations. When choosing the approximation methods we must bear in mind that the results depend, anyway, essentially on the estimation of the primary quantities $\varepsilon, \lambda, n$ etc., which is very much , a matter of taste" and which consequently cannot be accurate, so no great accuracy is needed in this approximation either.

I3. The theory of risk is developed on the basis of different basic assumptions. The older theory often took into account the pure random fluctuations only. It is, however, well-known, that the basic probabilities of the risks insured vary for different reasons. E.g. weather conditions can cause variations of this sort in some insurance branches. The general economic conditions have a noticecable influence upon most branches of non-life insurance. Some of these variations are themselves random fluctuations, which have different and mutually independent values from year to year. For them models, like that of Ammeter, are developed. Some other variations show long term trends and/or trends over periods of several years. For these the theory of risk is not developed as yet. Because these variations, however, are of essential significance, they must be taken into account in some way or other. We have used a rough method of building a different reserve $U$ both for pure random fluctuations and for the variations of basic probabilities. The latter is estimated directly from the past year's experience. If e.g. an economic depression period and other reasons can be expected, from earlier experience, to increase the frequency of claims by $20 \%$ and the maximum duration of the period is estimated to be 4 years, a reserve of $4.0,2 P=0.8 \mathrm{P}$ would be enough 
to cover the excess loss in such an event. Numbers like this can be deducted from the statistics of past decades.

I4. Concerning the pure random fluctuations different formulas are needed for different types of insurance business and also depending on the basic assumptions which are chosen. We will study only a couple of examples and start with the case $n=\infty$, i.e. the adjustment reserve $U$ is to be determined so that the probability of ruin gets a fixed value $\varepsilon$. The well-known formulas of the theory of risk are

$$
\varepsilon \approx e^{-R U}
$$

where the auxiliary constant $\mathrm{R}$ is arrived at as the positive root of the equation

$$
\int_{0}^{\infty} e^{R z} d s(z)=\mathrm{I}+(\mathrm{I}+\lambda) m R
$$

Here $s(z)$ is the distribution function of the size of the claims and $m$ is the mean value of them. Only positive risk sums are taken into account. To get the desired simple approximation we substitue $e^{R z}$ with the series

$$
\begin{aligned}
\mathrm{I}+R z & +\frac{(R z)^{2}}{2 !}+\ldots \text { and integrate: } \\
& \mathrm{I}+m R+\alpha_{2} \frac{R^{2}}{2 !}+\alpha_{3} \frac{R^{3}}{3 !}+\ldots=\mathrm{I}+(\mathrm{I}+\lambda) R m
\end{aligned}
$$

where

$$
\alpha_{i}=\int_{0}^{\infty} z^{i} d s \quad\left(\alpha_{1}=m\right)
$$

Now we assume that the company has a reinsurance which is so arranged that the maximum claim on net retention is $M$, i.e. $s(M)=\mathrm{I}$. It is easy to see then that

$$
m(k M)^{i-1} \leqq \alpha_{i} \leqq m M^{i-1} \quad(i=2,3, \ldots)
$$

where $k=\alpha_{2} / m M$. The equality is valid only when all risk sums $z$ are mutually equal and $=M$. Putting these approximations to the equations (8) and (9) we get after some elementary calculations

$$
k \frac{\ln \frac{\mathrm{I}}{\varepsilon}}{x_{\lambda}} M \leqq U \leqq \frac{\ln \frac{\mathrm{I}}{\varepsilon}}{x_{\lambda}} M
$$


Here $x_{\lambda}$ is the positive root of the equation

$$
e^{x}=\mathrm{I}+(\mathrm{I}+\lambda) x
$$

For small values of $\lambda$ we have $x_{\lambda} \approx 2 \lambda$

The upper limit of the formula (I2) is independent of the risk sum distribution $s(z)$. The coefficient $k$ depends on this distribution and it is different for different maximum net retentions $M$. If $M$ is not very large, $k$ seems to get values varying between 0.3 and $I$. Hence the approximation formula

$$
U=\frac{\ln \frac{\mathrm{I}}{\varepsilon}}{x_{\lambda}} M
$$

is in general satisfactory for our purpose.

If $\varepsilon=10^{-3}$ and $\lambda=0.05$ we get $U=69 M$ and for $\varepsilon=10^{-2}$ and $\lambda=0 . I$ we get $U=23 \mathrm{M}$.

I5. Because the very remote future is subject to many kinds of unknown changes a choice of some finit number of $n$ in the formula (7) instead of infinity may be reasonable. The company has also possibilities to counteract future fluctuations by suitable measures. E.g. by changing the reinsurance the risk balance can be improved if the company's development proves to be unfavourable. Saving in acquisition costs in cases of emergency has also been used with success as well as the amendment of the margin $\lambda$ (by effective selection of risks or increase of premiums, even though the possibility of the latter policy is often limited, owing to competition). On the other hand if a company acquires large reserves (the formula (8) provides in fact the increase towards infinity) the acquisition and other costs are soon increased or larger profits or a bonus are paid to share holders or policy holders. These aspects make it also advisable to take $n$ as finit.

For the number $n=\mathrm{I}$ suitable formulas are available. The numbers $n>$ I seem to lead to rather intricate computations, which are not convenient for every day practice. One way of procedure is to take $\mathrm{n}=\mathrm{I}$ and then apply some multiple of the reserve $\mathrm{U}$ so calculated.

Hence we can take in (7) $n=\mathrm{I}$. The problem is now to find some proper approximation for the distribution function $F(x)$ of the annual total amount of the claims. A simple formula is 


$$
F(x) \approx \Phi\left(\frac{x-P}{\sqrt{\alpha_{2} N}}\right)
$$

where $\Phi$ is the normal distribution, $\alpha_{2}$ is the moment defined in (Io) and $N$ is the expected number of claims. The validity of this approximation is shown by a number of numerically computed examples. It seems that this formula is not satisfactory if the risk sum distribution is rather heterogeneous and at the same time $N$ is small. If the risks are fairly homogeneous, which is the fact due to reinsurance, which is mostly in practice, and $N$ is not very small, the accuracy seems to be satisfactory, at least for the purpose in question.

We get now directly from (15), observing that $N=P / m$ and $x=$ $P(\mathrm{I}+\lambda)+U$ and using the same notations as in $\mathrm{nr}$. I4,

$$
y_{\varepsilon} \sqrt{k P} \bar{M}-\lambda P \leqq U \leqq y_{\varepsilon} \sqrt{P M}-\lambda P
$$

where $y_{\varepsilon}$ is the root of $\varepsilon=\Phi(-y)$.

The coefficient $k$, which characterized the risk sum distribution, appears here too.

In the formula (I6) the limits of $U$ are functions of $P$. They have the maximums

$$
\left.U=\frac{y^{2} k}{4^{\lambda}} M \quad \text { (for the upper limit } k=\mathrm{I}\right)
$$

which is very similar to the formula (I4). We have for $k=\mathrm{I}, \varepsilon=$ $\mathrm{IO}^{-3}$ and $\lambda=0.05, U=48 \mathrm{M}$ and for $k=0.7, \varepsilon=1 \mathrm{I}^{-2}$ and $\lambda=$ o.I, $U=$ Io $M$.

I6. We can now conclude that adding to the formula (I6) the provision mentioned in $\mathrm{nr}$. I3 we get a standard formula of the type

$$
U=a P+b \sqrt{P M}
$$

where the constants $\mathrm{a}$ and $\mathrm{b}$ can be fixed, choosing the assumptions concerning $\varepsilon, \lambda$, $\mathrm{n}$ etc. in a suitable way. 